\title{
Liddle's Syndrome Associated with Acromegaly due to Enhanced Renal and Extrarenal ENaC Channel Activity: The First Case Report that Explained this Rare Association
}

\section{Juna Musa ${ }^{1 *}$, Jovan Basho ${ }^{2}$, Florian Toti $^{3}$, Suela Mumajesi ${ }^{4}$, Mohammed Badi ${ }^{5}$, Adi Abduli ${ }^{6}$, Loran Rakovica ${ }^{7}$ and Ali Guy ${ }^{8}$}

${ }^{1}$ Postdoctoral Research Fellow, Department of Surgery, Mayo Clinic, Minnesota, USA ${ }^{2}$ Department of Gastro-Hepatology, University of Hospital Center Mother Teresa, Tirana, Albania

${ }^{3}$ Department of Endocrinology, University of Hospital Center Mother Teresa, Tirana, Albania

${ }^{4}$ Department of Nephrology and Dialysis, University of Hospital Center Mother

Teresa, Tirana, Albania

${ }^{5}$ Postdoctoral Research Fellow, Department of Neurology, Mayo Clinic, Jacksonville,

Florida, USA

${ }^{6}$ Department of Emergency Care, University of Hospital Center Mother Teresa,

Tirana, Albania

${ }^{7}$ Faculty of Medicine, University of Prishtina, Kosovo

${ }^{8}$ Department of Physical Medicine and Rehabilitation, School of Medicine - NYU

Medical Center, New York, USA

*Corresponding Author: Juna Musa, Postdoctoral Research Fellow, Department of Surgery, Mayo Clinic, Minnesota, USA.
Received: April 12, 2020

Published: April 27, 2020

(C) All rights are reserved by Juna Musa., et al.

\begin{abstract}
Liddle's syndrome is a rare genetic autosomal dominant disease affecting the activity of the epithelial sodium channels (ENaC) or the amiloride-sensitive sodium channels, which leads the kidneys to excrete potassium but retain too much sodium and water causing early, frequent and severe, high blood pressure associated with low plasma renin, metabolic alkalosis and normal to low levels of aldosterone [1,2]. Disease-associated mutations either activate the channel directly or abrogate aldosterone-inhibited retrieval of $\mathrm{ENaC}$ subunits from the plasma membrane [3]. The end result of these mutations is increased expression of activated $\mathrm{ENaC}$ channels at the plasma membrane of principal cells which manifests as severe early onset hypertension with hypokalemia unresponsive to spironolactone [4]. We observed an isolated case of a Liddle's syndrome in a 23-year-old man who presented with signs of acromegaly. The patient initially presented to the emergency room with palpitations, weakness of the extremities and a three-year history of low potassium levels and uncontrolled hypertension. Six months later he was admitted again to the hospital with signs of hypokalemia, including palpitations, fatigue and weakness. Upon further evaluation, he was found to have low renin and aldosterone levels. Physical examination revealed mild diastolic hypertension, acromegalic features, weight gain, excessive sweating, widening of both hands and feet [5]. Laboratory results revealed increased levels of GH and IGF-1-a. After oral administration of oral glucose levels of IGF-1 remained elevated. MR imaging of the brain revealed a $1.3 \times 1.2 \times 0.7 \mathrm{~cm}$ pituitary adenoma. A diagnosis of acromegaly and Liddle syndrome was made. Coexisting acromegaly and Liddle syndrome are reported together only in rare cases and it can be explained due to enhanced renal and extrarenal ENaC activity.

Keywords: Liddle's Syndrome; Epithelial Sodium Channels (ENaC); Acromegaly
\end{abstract}

\section{Introduction}

Liddle syndrome is a rare genetic condition, characterized by early severe high blood pressure associated with low plasma renin activity, metabolic alkalosis, low blood potassium and normal to low levels of aldosterone that develops at an early age [6]. Mutation in the $S C N N 1 B$ or $S C N N 1 G$ genes are responsible for this syndrome [7].

The incidence of Acromegaly is 3 cases per million per year. Acromegaly occurs due to excessive growth hormone (GH) secretion, with most cases caused by the presence of a benign pituitary ad- enoma. The body dysmorphic features in Acromegaly is due to the effects of GH/IGF1 on skeletal growth and in soft tissue infiltration, while some patients may present with visual impairment due to encroachment of the optic chiasm. Acromegaly is diagnosed by the presence of pituitary adenoma, signs of atypical clinical features and high levels of GH and IGF-1.

It is very important to check patients with Acromegaly after visual assessment due to the proximity of adenoma with the optic chiasm. 


\section{Treatment}

The choice of treatment in Acromegaly due to pituitary macro adenoma is trans-sphenoidal resection surgery or medical therapy, of which octerotide is the most commonly used.

\section{Case Report}

A 23-year-old Caucasian male, presented to the emergency department with fatigue, weakness, leg cramps, palpitation, polyuria, nocturnal enuresis and polydipsia and uncontrolled hypertension (200/100 $\mathrm{mmHg}$ ). The patient also reported weight gain, enlargement in his hands and feet sizes, and changes in his voice. An abdominal CT scan revealed splenomegaly, hepatic and splenic lesions. Family history was non-contributory for any of his symptoms/signs. Past medical history was positive for visceral leishmaniosis, polyuria and polydipsia and negative for convulsion, visual function impairment, cardiac diseases or diabetes mellitus.

Physical examination showed coarse and prominent facial features that developed over a course of several years accompanied with enlargement of hands and feet, according to the patient. Coarse facial features included enlarged frontal and nasal bone, slightly prominent supraorbital ridges, large ears and fleshy nose. He also had thickened lips, widening of teeth spaces, especially front upper incisors, macroglossia and prognathism. The skin was thickened, oily and malodorous, with no focal pigmentation, no skin tags, no pallor, cyanosis, and icterus or clubbing. His hands and feet seemed to be enlarged with no signs of edema, skeletal deformation, pectus carinatum (pigeon chest) and scoliosis, with no musculoskeletal pain or nerve impingement syndrome. His height was $1.87 \mathrm{~m}$ and his weight was $78 \mathrm{~kg}$ with a calculated BMI of 22.3 $\mathrm{kg} / \mathrm{m}^{2}$ (normal). He had somehow a deep, husky voice and snored during sleep, but there was no daytime sleepiness or other signs of obstructive sleep apnea.

MRI Brain shows 1 x 1.5 x $1.7 \mathrm{~cm}$ well defined lesion pituitary macroadenoma.

Abdominal MRI showed two splenic lesions (5 x 6 and $4 \times 4$ $\mathrm{cm}$ of diameter) compatible with hamartomas. Three lesions of the liver ( $4 \times 3,3 \times 2$ and $2 \times 2 \mathrm{~cm}$ of diameter) compatible with hemangiomas, the patient refused biopsy of these lesions so no further investigations were done on this regard. The right kidney was enlarged $(16.5 \mathrm{~cm}$ of diameter and was located under the right diaphragm). The left kidney had normal size but was positioned lower.

Past medical history: Seventeen years ago, when he was 6 years old, he was diagnosed with visceral leishmaniasis for which he was treated with glucantime (Meglumine antimoniate).

For several years, the patient has been suffering from resistant and uncontrollable high blood pressure (up to 200/100 $\mathrm{mmHg}$ ), currently being managed with multiple antihypertensive drugs (including spironolactone started recently).

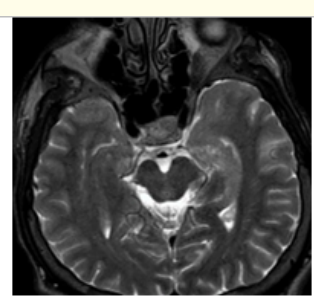

Figure 1

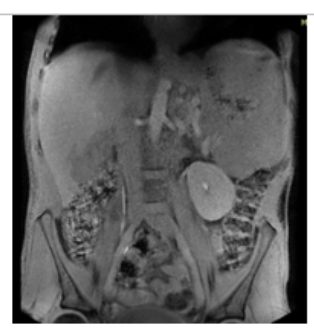

Figure 3

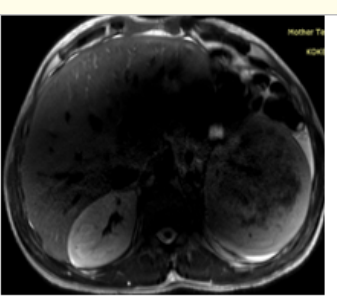

Figure 2

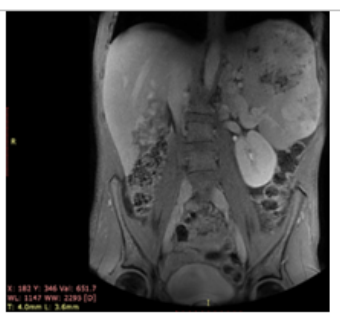

Figure 4
There was no other clue in his medical history to suggest previous diuretic use or licorice consumption.

Family history: Both of his parents were healthy without any disease and his 19-year-old brother has a normal appearance, without signs and symptoms of any disease, including hypertension.

\section{Discussion}

Liddle syndrome is characterized by hypertension, hypokalemic metabolic alkalosis, low plasma renin activity (PRA) and low aldosterone levels. Clinically patients resemble those with mineralocorticoid excess and hyperaldosteronism. Hallmark of this disorder is the finding of markedly suppressed serum aldosterone levels and the lack of response to administration of mineralocorticoid receptor blocker spironolactone. The disorder is localized to a specific mutation in the epithelial $\mathrm{Na}^{+}$channel (ENaC) located in the collecting duct of the kidney. In the collecting duct and connecting tubule, the epithelial sodium channel (ENaC) plays a critical role in extracellular $\mathrm{Na}^{+}$and volume homeostasis, hence in control of blood pressure [8].

This amiloride-sensitive epithelial sodium channel, composed of three homologous subunit ( $\alpha \mathrm{ENac}, \beta \mathrm{ENaC}$ and $\gamma \mathrm{ENac}$ encoded by SCNN1A, SCNN1B and SCNN1G) forms a pathway for $\mathrm{Na}^{+}$reabsorption across a variety of epithelia. The $\beta$-subunit alone can induce low levels of sodium channel activity, while the $\beta$ and $\gamma$ subunits have been reported to modify the channel activity $[9,10]$. All reported mutations found in patients with Liddle syndrome are either missense or frameshift mutations that destroy the conserved proline-rich PY motif (PPXY sequence) located at the C-terminal ends of $\beta$ and $\gamma$ subunits of ENaC [11,12]. Hormones, including aldosterone and insulin, increase $\mathrm{ENaC}$ activity, in part phosphatidylinositide 3-OH kinase (PI3-K) signaling [13]. Recent studies reveal a close spatiotemporal coupling between PI3-K signaling and $\mathrm{ENaC}$ activity with phospholipid product of this kinase, which in some cases can directly bind the $\mathrm{ENaC}$ channel and increasing 
its opening probability. IGF-1 was found to increase the $\mathrm{Na}^{+}$reabsorption across the mpkCCDc14 principal monolayers. Moreover, inhibition of PI3-K signaling rapidly decreased $\mathrm{Na}^{+}$reabsorption and ENaC activity in mpkCCDc14 cells that were treated with corticosteroid and IGF-1 - these changes demonstrate a right spatiotemporal coupling between ENaC activity and PI3-K/PI(3,4,5)P3 signaling within the membrane. Insulin and insulin-like growth factor 1 (IGF-1) may play a more important role than thought before in the regulation of sodium balance by increasing basal and aldosterone-stimulated transepithelial transport in aldosteronesensitive distal nephron [14].

Systematic review of the reported cases revealed that hypertension is present in $92.4 \%$ of the patients with Liddle syndrome, hypokalemia (defined as serum $\mathrm{K}^{+}<3.5 \mathrm{mmol} / \mathrm{L}$ ) in $71.8 \%$ and hypoaldosteronemia (defined as serum aldosterone $<5 \mathrm{ng} / \mathrm{dL}$ ) in $58.2 \%$ of the cases [15].

It is likely that both environmental and genetic factors, including $\mathrm{Na}^{+}$intake and polymorphisms in genes involved in $\mathrm{Na}^{+}$handling could influence the phenotypic manifestation of the disease.

Our patient presented with a classical clinical picture of Liddle Syndrome, he presented with early onset, uncontrolled hypertension, polyuria, nocturia, polydipsia and severe weakness due to hypokalemia, and laboratory test also revealed metabolic alkalosis together with hyporeninemia and hypoaldosteronemia.

Fortunately, the diagnosis of Liddle syndrome was made in time and treatment was initiated before installment of severe or fatal complications of hypertension or hypokalemia [16].

Firstly, we treated the patient with high dose of I.V. potassium chloride (120 mEq/day), 1.5 gram potassium chloride orally, Triamterene $200 \mathrm{mg} /$ day and antihypertensive drugs. The response was immediate and excellent, further supporting our diagnosis. Daily diuresis was immediately reduced to $2-2.51$ and the patient did not have the feeling of thirst any more. The patient was also advised to follow a low salt diet ( $2 \mathrm{~g} \mathrm{NaCl} /$ day) and regular monitoring of his electrolytes (especially Potassium levels). Considering the frequency of early-onset hypertension and severity of correlated complications, a well-timed diagnosis of Liddle Syndrome is very important in order to administer the proper therapy.

There were no signs of cardiac changes due to hypertension - echocardiography was normal, with no evidence of LV hypertrophy or dilation) no signs of renal damage - creatinine and GFR were within normal range) no sign of hypertensive retinopathy - fundoscopic examination was normal as were visual acuity and visual fields).

In our case we use the exceptional criteria to find the right diagnoses.
In a patient with hypertension and hypokalemic alkalosis, primary aldosteronism, Cushing's syndrome, pheochromocytoma, renovascular hypertension, essential hypertension with diuretic use, some forms of congenital adrenal hyperplasia, Liddle syndrome and syndrome of apparent mineralocorticoid excess have to be considered as differential diagnoses. Low levels of rennin, aldosterone and normal values of serum cortisol, ACTH and urinary cortisol and catecholamines, together with normal abdominal CT/MRI excluded Primary aldosteronism, Cushing's syndrome, Pheochromocytoma, Reno-Vascular Hypertension, Congenital Adrenal Hyperplasia. Apparent mineralocorticoid excess leads to high cortisol due to deficiency of $11 \beta$ hydroxysteroid dehydrogenase 2 (which inactivates cortisol turning it to cortisone), this elevated cortisol binds to the cytosol mineralocorticoid receptor, creating a state of apparent mineralocorticoid excess leading to hypokalemic hypertension. But normal levels of cortisol and ACTH, and the lack of response to spironolactone, excluded this diagnosis.

Liddle syndrome is usually associated with a positive family history, although our case was a sporadic one, and the lack of family history cannot be used to exclude this diagnosis [17]. The most reliable testing for Liddle syndrome is genetic testing to confirm the mutation in ENaC subunits genes, but genetic testing is not necessary to make the diagnosis.

Low urine sodium ( $<20 \mathrm{mEq}$, or $20 \mathrm{mmol} / \mathrm{L}$ ), low plasma renin and aldosterone levels, and response to empiric treatment (with $\mathrm{ENaC}$ blockers like amiloride and triamterene) usually are considered sufficient to confirm the diagnosis. All of these criteria were met in our patient, confirming the diagnosis of LS, and the definite diagnosis was finally achieved through genetic testing.

What complicated the diagnosis was the co-existence of acromegalic features.

Recent studies show that excess GH/IGF-1 in active acromegaly increases activity of ENaC leading to more sodium retention, volume expansion and hypertension [18]. The generalized edema together with increased collagen production by connective tissue and GAG deposits is thought to be responsible for generalized soft tissue infiltration and expansion. The increase in extracellular fluid may contribute to acromegaly patients' higher prevalence of arterial hypertension and cardiomyopathy, two major prognostic factors of excess mortality.

The effect of the GH excess on renal and extra renal epithelial sodium channel ENac activity and the defined pathophysiological mechanism is still unclear. Further investigations are needed for evaluation. Some experimental studies in mice and rats revealed that $\mathrm{GH}$ has a direct stimulating effect on epithelial sodium channel ENaC dependent sodium transport. 


\section{Conclusion}

The specific treatment for Liddle Syndrome is by ENaC channel blockers, like the $\mathrm{K}^{+}$sparing diuretics amiloride and triamterene. They not only treat hypertension but they also normalize the typical biochemical alterations (hypokalemia, suppressed PRA and low aldosterone level).

Neither hypertension nor hypokalemia improve in response to spironolactone (since activation of the mineralocorticoid receptor is not implicated in $\mathrm{Na}^{+}$reabsorption) and this might represent an additional clinical criterion to suspect Liddle syndrome.

Taking into consideration the fact that there is a competition between $\mathrm{Na}^{+}$and $\mathrm{ENaC}$ blockers and low sodium diet (2g NaCl/day) should be recommended for the patient.

Next $\mathrm{K}^{+}$supplementation should be initiated first I.V. potassium to correct severe and dangerous hypokalemia.

Continuous monitoring of electrolytes is highly recommended although hyperkalemia is rare in a patient with normal renal function (as in our patient) and when potassium intake is not excessive.

And finally considering the chronic, insidious nature of acromegaly (dysmorphic acromegalic changes occur very gradually over a period of years without the patient or their close relatives being aware of or noticing these changes, and eventually seek medical help about them), and the dangerous long term complications of active acromegaly (like cardiovascular disease, respiratory disorders, DM, increased risk of malignancy etc.), it is of utmost importance to have regular follow-ups with an endocrinologist and regular measurements of GH/IGF-1 levels.

All the above measurement were undertaken in our patient and fortunately there was significant improvement of his clinical and biochemical changes.

\section{Bibliography}

1. Biff F Palmer., et al. "Liddle's Syndrome". The American Journal of Medicine 104 (1998): 301-309.

2. Botero-Velez M., et al. "Liddle's syndrome revisited: disorder of sodium reabsorption in the distal tubule". The New England Journal of Medicine 330 (1994): 178-181.

3. Snyder PM., et al. "Mechanism by which Liddle's syndrome mutations increase activity of a human epithelial $\mathrm{Na}+$ channel”. Cell 83 (1995): 969-978.

4. Jameson, Fauci., et al. "Harrison's Principle of Internal Medicine $20^{\text {th }}$ edition".
5. Peter Kamenicky., et al. "Body Fluid Expansion in Acromegaly Is Related to Enhanced Epithelial Sodium Channel (ENaC) Activity". Journal of Clinical Endocrinology and Metabolism 96.7 (2011): 2127-2135.

6. Zennaro MC., et al. "Inherited forms of mineralocorticoid hypertension". Best Practice and Research Clinical Endocrinology and Metabolism 29 (2015): 633-645.

7. Yibo Wang., et al. "A novel epithelial sodium channel $\gamma$-subunit de novo frameshift mutation leads to Liddle syndrome". Clinical Endocrinology 67 (2007): 801-804.

8. Marko Bertog., et al. "Aldosterone responsiveness of the epithelial sodium channel $(\mathrm{ENaC})$ in colon is increased in a mouse model for Liddle's syndrome". The Journal of Physiology 586.2 (2008): 459-475.

9. Sawathiparnich P., et al. "A novel mutation in the ß-subunit of the epithelial sodium channel gene (SCNN1B) in a Thai family with Liddle's syndrome". Journal of Pediatric Endocrinology and Metabolism 22 (2009): 85-89.

10. Hansson JH., et al. "A de novo missense mutation of the $§$ subunit of the epithelial sodium channel causes hypertension and Liddle syndrome, identifying a proline-rich segment critical for regulation of channel activity". Proceedings of the National Academy of Sciences of the United States of America 92 (1995): 11495-11499.

11. Wang Y., et al. "A novel epithelial sodium channel $\gamma$-subunit de novo frameshift mutation leads to Liddle syndrome". Journal of Clinical Endocrinology 67 (2007): 801-804.

12. Kamide K., et al. "Six missense mutations of the epithelial sodium channel beta and gamma subunits in Japanese hypertensives". Hypertension Research 27.5 (2004): 333-338.

13. Alexander Staruschenko., et al. "Acute Regulation of the Epithelial Na Channel by Phosphatidylinositide 3-OH Kinase Signaling in Native Collecting Duct Principal Cells". Journal of the American Society of Nephrology 18 (2007): 1652-1661.

14. E Gonzalez-Rodriguez., et al. "IGF-1 vs insulin: Respective roles in modulating sodium transport via the PI-3 kinase/Sgk1 pathway in a cortical collecting duct cell line". Kidney International 71 (2007): 116-125.

15. Martina Tetti., et al. "Liddle Syndrome: Review of the Literature and Description of a New Case". International Journal of Molecular Sciences 19.3 (2018): 812.

16. Mulatero P., et al. "Diagnosis and treatment of low-renin hypertension". Journal of Clinical Endocrinology 67 (2007): 324-334.

17. Yamashita Y., et al. "Two sporadic cases of Liddle's syndrome caused by de novo ENaC mutations". American Journal of Kidney Diseases 37 (2001): 499-504. 
18. Peter Kamenicky., et al. "Epithelial Sodium Channel Is a Key Mediator of Growth Hormone-Induced Sodium Retention in Acromegaly". Endocrinology 149.7 (2008): 3294-3305.

\section{Assets from publication with us}

- Prompt Acknowledgement after receiving the article

- Thorough Double blinded peer review

- Rapid Publication

- Issue of Publication Certificate

- High visibility of your Published work Website: https://www.actascientific.com/ Submit Article: https://www.actascientific.com/submission.php Email us: editor@actascientific.com

Contact us: +919182824667 